\title{
ON WEAK REVERSE INTEGRAL INEQUALITIES FOR MEAN OSCILLATIONS
}

\author{
MICHELANGELO FRANCIOSI
}

(Communicated by Barbara L. Keyfitz)

\begin{abstract}
We prove that if $f$ verifies a reverse Hölder inequality with exponent $p, 1<p<+\infty$, then $\left(M f+f^{\sharp}\right)^{p}$ is a $A_{1}$-weight of Muckenhoupt, where $M f$ is the Hardy-Littlewood maximal function and $f^{\sharp}$ the FeffermanStein maximal function.
\end{abstract}

\section{INTRODUCTION}

Reverse integral inequalities occur in many fields of analysis such as quasiconformal mappings, weighted Sobolev imbedding theorems, and regularity of solutions of partial differential equations (see $[3,4,7,14]$ and many others).

A classical result about functions verifying reverse integral inequalities or related inequalities is extraintegrability. A prototype of these results is the famous higher integrability result of Gehring about the derivatives of the components of a quasiconformal mapping, published in 1973 [12]. See also [3, 4, 16, 9, 14].

Probably the first considered and simplest reverse integral (mean) inequality is the following $[15,1972]$ :

$$
f_{Q} g d x \leq D \text { ess inf } g
$$

where the constant $D \geq 1$ is the same for all the parallel subcubes of a fixed cube $Q_{0} \subset R^{n}$ and $g$ is a function that is positive in $Q_{0}$. A function verifying $\left(A_{1}\right)$ is called an $A_{1}$-weight of Muckenhoupt. In [15] it is proved that any $A_{1}$-weight belongs to $L^{r}\left(Q_{0}\right)$ for $1 \leq r<s$ and $s$ depending only on $n$ and $D$.

In this paper we consider weak reverse integral inequalities in a weighted case (see $[4,5,14]$, e.g.), that is reverse integral inequalities with different supports. Such inequalities are much more useful in the applications to partial differential equations than the reverse integral inequalities $[4,7]$.

Received by the editors March 5, 1990 and, in revised form, July 18, 1990.

1980 Mathematics Subject Classification (1985 Revision). Primary 42B25; Secondary 46E30.

Key words and phrases. Reverse inequalities, Mean oscillations, maximal functions, $A_{p}$-weights.

This work was partially supported by a national project of the Italian Ministero della Pubblica Istruzione and by G.N.A.F.A.-C.N.R. 
In particular we consider inequalities like

$$
\left(f_{\sigma Q}\left|f-f_{\sigma Q} f d \mu\right|^{p} d \mu\right)^{1 / p} \leq K f_{Q}\left|f-f_{Q} f d \mu\right| d \mu,
$$

where $K>1$ is independent on the parallel subcube $Q$ of $Q_{0}, 0<\sigma \leq$ $1, p>1$ and $\mu$ is a doubling measure with density (see $\S 1$ for more details).

We prove that if (WR) holds, then the function $\left(M f+f^{\sharp}\right)^{p}$ is an $A_{1^{-}}$weight. Here $M f$ is the Hardy-Littlewood local maximal function and $f^{\sharp}$ is the Fefferman-Stein local maximal function (see [1] e.g.). From this, using some rearrangement techniques, a weighted version of the Herz theorem, [7, 13, 16], and the Muckenhoupt lemma, we deduce a higher integrability result for any $f$ verifying condition (WR).

We emphasize that the main step in obtaining higher integrability from (WR) is in stating that the Muckenhoupt lemma [15] holds for $\left(M f+f^{\sharp}\right)^{p}$. This approach is substantially different (see also $[5,9,10,16,17]$ for similar cases) from many others available in literature $[3,4,6,10,12,14,17,19]$.

\section{MAIN RESUlt}

Let $Q_{0}$ be a cube of $R^{n}$ whose edges are parallel to the axes. All the subcubes of $Q_{0}$ that are considered, have the edges that are parallel to the axes.

Let $w$ be a nonnegative function that is locally summable in $R^{n}$, and for any measurable subset $E$ of $R^{n}$ consider the measure

$$
\mu(E)=\int_{E} w(x) d x .
$$

For any $\delta>0$ and any cube $Q \subseteq Q_{0}$ we set $\delta Q$ for the cube concentric with $Q$ and such that $|\delta Q|=\delta^{n}|Q|$, where $|E|$ indicates the Lebesgue measure of the measurable set $E$.

We say that the weight function $w$ satisfies the strong doubling condition $([3,16]$ e.g. $)$ if the inequality

$$
\mu(\delta Q) \leq d \mu(Q)
$$

holds for some positive constant $d$ depending only on $\delta$ and $n$ (and independent on the cube $Q \subseteq Q_{0}$ ).

For any $f \in L_{\text {loc }}^{1}\left(Q_{0}\right)$ and any cube $\bar{Q} \subseteq Q_{0}$ we consider the local maximal function of Hardy-Littlewood:

$$
M f(x)=\sup _{\substack{x \in Q \\ Q \subset \bar{Q}}} f_{Q}|f(x)| d \mu, \quad x \in \bar{Q},
$$

and the local maximal function of Fefferman-Stein:

$$
f^{\sharp}(x)=\sup _{\substack{x \in Q \\ Q \subset \bar{Q}}} f_{Q}\left|f(x)-f_{Q} f d \mu\right| d \mu, \quad x \in \bar{Q},
$$


where for any $h \in L_{\text {loc }}^{1}\left(Q_{0}\right), E \subseteq Q_{0}$, we set $f_{E} h(x) d \mu=(1 / \mu(E)) \int_{E} h(x) d \mu$. We say that a nonnegative function $h \in L_{\mathrm{loc}}^{1}\left(Q_{0}\right)$ is an $A_{1}$-weight of Muckenhoupt if [15]

$$
f_{Q} h(x) d \mu \leq D \text { ess inf } h(x)
$$

for some constant $D>1$ independent on the cube $Q \subset Q_{0}$.

We prove the following:

Lemma 1.1. Let $f$ be a nonnegative function belonging to $L_{\text {loc }}^{1}\left(Q_{0}\right), 0<\sigma \leq 1$. We suppose that $w$ verifies condition (D) and that

$$
\left(f_{\sigma Q}\left|f-f_{\sigma Q} f d \mu\right|^{p} d \mu\right)^{1 / p} \leq K f_{Q}\left|f-f_{Q} f d \mu\right| d \mu,
$$

where $p>1$, with $K$ positive constant and independent on the cube $Q \subseteq Q_{0}$. Then the function $\left(M f+f^{\sharp}\right)^{p}$ is an $A_{1}$-weight of Muckenhoupt.

Proof. Fix $Q \leq Q_{0}$ such that $Q / \sigma \subset Q_{0}, Q^{\prime} \subset \sigma^{2} Q$. We pick five cubes in this way to stay in $Q_{0}$. For a fixed $z \in Q^{\prime}$, let $Q \subset \bar{Q}$ be such that $z \in Q$. Suppose $0<\sigma \leq \frac{1}{2}$, if $\frac{1}{2}<\sigma \leq 1$ we proceed similarly. From the proof it will follow that the two cases $Q \subseteq Q^{\prime} / \sigma, Q \nsubseteq Q^{\prime} / \sigma$ must be treated separately. If $Q \subseteq Q^{\prime} / \sigma$ then

$$
\begin{aligned}
f_{Q}\left|f(x)-f_{Q} f d \mu\right| d \mu & =f_{Q}\left|f \chi_{Q^{\prime} / \sigma}-f_{Q} f \chi_{Q^{\prime} / \sigma} d \mu\right| d \mu \\
& \leq\left(f \chi_{Q^{\prime} / \sigma}\right)^{\sharp}(z),
\end{aligned}
$$

where $\chi_{E}$ is the characteristic function of $E$. In the same way

$$
\begin{aligned}
f_{Q} f(x) d \mu & =f_{Q}\left(f \chi_{Q^{\prime} / \sigma}\right)(x) d \mu \\
& \leq f_{Q}\left|f \chi_{Q^{\prime} / \sigma}-\left(f_{Q^{\prime} / \sigma} f \chi_{Q^{\prime} / \sigma} d \mu\right) \chi_{Q^{\prime} / \sigma}\right| d \mu+f_{Q^{\prime} / \sigma} f \chi_{Q^{\prime} / \sigma}(x) d \mu \\
& \leq M\left(\left(f-f_{Q^{\prime} / \sigma} f \chi_{Q^{\prime} / \sigma} d \mu\right) \chi_{Q^{\prime} / \sigma}\right)(z)+\operatorname{ess} \inf _{Q^{\prime} / \sigma} M\left(f \chi_{Q^{\prime} / \sigma}\right)(z) .
\end{aligned}
$$

If $Q \nsubseteq Q^{\prime} / \sigma$ it is easy to see that there exists a cube $\widetilde{Q}$ such that $\widetilde{Q} \supseteq$ $Q \cup Q^{\prime}, \widetilde{Q} \subset Q_{0},|\widetilde{Q}| \leq 3^{n}|Q|$. Using condition (D) the existence of a positive constant $d$ independent on $Q \subset Q_{0}$ such that $\mu(\widetilde{Q}) \leq d \mu(Q)$ is clear.

We then have

$$
\begin{aligned}
f_{Q}\left|f(x)-f_{Q} f d \mu\right| d \mu & \leq d f_{\widetilde{Q}}\left|f \chi_{Q}-f_{\widetilde{Q} \cap Q} f \chi_{Q} d \mu\right| d \mu \\
& \leq d \sup _{z \in C}^{\prime} f_{C}\left|f \chi_{Q}-f_{C \cap Q} f \chi_{Q} d \mu\right| d \mu,
\end{aligned}
$$


where $\sup ^{\prime}$ is extended to all cubes $C$ containing $z$ and such that $C \cap Q$ is a cube. We deduce

$$
\begin{aligned}
f_{Q}\left|f(x)-f_{Q} f d \mu\right| d \mu \leq & d \sup _{z \in C}^{\prime} f_{C \cap Q}\left|f \chi_{Q}-f_{C \cap Q} f \chi_{Q} d \mu\right| d \mu \\
& +d \sup _{z \in C}^{\prime} f_{C \cap Q} f \chi_{Q} d \mu \\
\leq & d f^{\sharp}(z)+d M f(z) \\
\leq & d \inf _{z \in Q} f^{\sharp}(z)+d \inf _{z \in Q} M f(z),
\end{aligned}
$$

because $\widetilde{Q} \supset Q^{\prime}$. Otherwise, if $z \notin Q$, there exists $z^{\prime} \in Q$ such that

$$
f_{C \cap Q}\left|f-f_{C \cap Q} f d \mu\right| d \mu \leq f^{\sharp}\left(z^{\prime}\right), \quad f_{C \cap Q} f d \mu \leq M f\left(z^{\prime}\right)
$$

and then

$$
f_{Q}\left|f-f_{Q} f d \mu\right| d \mu \leq d \inf _{z \in Q^{\prime}} f^{\sharp}(z)+d \inf _{z \in Q^{\prime}} M f(z) .
$$

If $Q \nsubseteq Q^{\prime} / \sigma$, obviously

$$
f_{Q} f d \mu \leq d f_{\widetilde{Q}} f d \mu \leq d \inf _{z \in Q^{\prime}} M f(z) .
$$

Using Hardy-Littlewood maximal inequality [1] we have

$$
\begin{gathered}
\int_{Q^{\prime} / \sigma}\left(M\left(\left(f \chi_{Q^{\prime} / \sigma}-f_{Q^{\prime} / \sigma} f \chi_{Q^{\prime} / \sigma} d \mu\right)(x)\right)\right)^{p} d \mu \\
\leq c_{0} \int_{Q^{\prime} / \sigma}\left|f \chi_{Q^{\prime} / \sigma}-f_{Q^{\prime} / \sigma} f \chi_{Q^{\prime} / \sigma} d \mu\right|^{p} d \mu,
\end{gathered}
$$

where $c_{0}$ is a constant depending only on $n$ and $p$.

From (1.1)-(1.4) we obtain

$$
\begin{aligned}
\left(f^{\sharp}(z)+M f(z)\right)^{p} \leq c_{1}( & \left|\left(f \chi_{Q^{\prime} / \sigma}\right)^{\sharp}(z)\right|^{p}+d^{p} \inf _{z \in Q^{\prime}}|M f(z)|^{p} \\
& +\left|M\left(\left(f \chi_{Q^{\prime} / \sigma}-f_{Q^{\prime} / \sigma} f \chi_{Q^{\prime} / \sigma} d \mu\right) \chi_{Q^{\prime} / \sigma}\right)(z)\right|^{p} \\
& \left.+d^{p} \inf _{z \in Q^{\prime}}\left|f^{\sharp}(z)\right|^{p}\right),
\end{aligned}
$$

where $c_{1}$ is a constant depending only on $p$. Dividing by $\mu\left(Q^{\prime}\right)$, integrating 
over $Q^{\prime} / \sigma$, and using (1.5) we obtain

$$
\begin{aligned}
f_{Q^{\prime}}\left|f^{\sharp}(z)+M f(z)\right|^{p} d \mu \leq & c_{1} d_{1} f_{Q^{\prime} / \sigma}\left|f\left(\chi_{Q^{\prime} / \sigma}\right)^{\sharp}(z)\right|^{p} d \mu \\
& +c_{1} d^{p} \inf _{z \in Q^{\prime}}|M f(z)|^{p}+c_{1} d^{p} \inf _{z \in Q^{\prime}}\left|f^{\sharp}(z)\right|^{p} \\
& +c_{0} c_{1} d_{1} f_{Q^{\prime} / \sigma}\left|f \chi_{Q^{\prime} / \sigma}-f_{Q^{\prime} / \sigma} f \chi_{Q^{\prime} / \sigma} d \mu\right|^{p} d \mu,
\end{aligned}
$$

where $d_{1}$ is the constant appearing in $(D)$ for $\delta=1 / \sigma$.

On the other hand

$$
\begin{aligned}
f_{Q^{\prime} / \sigma} & \left|\left(f \chi_{Q^{\prime} / \sigma}\right)^{\sharp}(z)\right|^{p} d \mu \\
= & f_{Q^{\prime} / \sigma}\left|\left(f \chi_{Q^{\prime} / \sigma}-\left(f_{Q^{\prime} / \sigma} f \chi_{Q^{\prime} / \sigma} d \mu\right) \chi_{Q^{\prime} / \sigma}\right)^{\sharp}(z)\right|^{p} d \mu \\
\leq & 2 f_{Q^{\prime} / \sigma}\left|M\left(f \chi_{Q^{\prime} / \sigma}-\left(f_{Q^{\prime} / \sigma} f \chi_{Q^{\prime} / \sigma} d \mu\right) \chi_{Q^{\prime} / \sigma}\right)(z)\right|^{p} d \mu \\
& \leq 2 f_{Q^{\prime} / \sigma}\left|f \chi_{Q^{\prime} / \sigma}-f_{Q^{\prime} / \sigma} f \chi_{Q^{\prime} / \sigma} d \mu\right|^{p} d \mu .
\end{aligned}
$$

Then from (1.6) and (1.7)

$$
\begin{aligned}
f_{Q^{\prime}}\left|\left(f^{\sharp}+M f\right)(z)\right|^{p} d \mu \leq & c_{1} d^{p} \inf _{z \in Q^{\prime}}\left|\left(f^{\sharp}+M f\right)(z)\right|^{p} \\
& +c_{2} f_{Q^{\prime} / \sigma}\left|f(z)-f_{Q^{\prime} / \sigma} f d \mu\right|^{p} d \mu,
\end{aligned}
$$

where $c_{2}$ is a constant depending only on $n, p$, and $w$.

Now we use condition (WR) to obtain

$$
\begin{aligned}
f_{Q^{\prime}}\left|\left(f^{\sharp}+M f\right)(z)\right|^{p} d \mu \leq & c_{1} d^{p} \inf _{z \in Q^{\prime}}\left|\left(f^{\sharp}+M f\right)(z)\right|^{p} \\
& +c_{2} K\left(f_{Q^{\prime} / \sigma^{2}}\left|f-f_{Q^{\prime} / \sigma^{2}} f d \mu\right| d \mu\right)^{p} \\
\leq & c_{1} d^{p} \inf _{z \in Q^{\prime}}\left|\left(f^{\sharp}+M f\right)(z)\right|^{p}+c_{2} K \inf _{z \in Q^{\prime}}\left|f^{\sharp}(z)\right|^{p},
\end{aligned}
$$

because $Q^{\prime} \subset Q^{\prime} / \sigma^{2}$. The lemma is proved.

We remark that the proof of Lemma 1.1 does not depend on any covering lemma.

\section{HighER INTEGRABILITY RESULT}

In this section we deduce from Lemma 1.1 a higher integrability result for functions satisfying condition (WR) . 
We recall the following Muckenhoupt lemma [13, p. 213] on higher integrability from reverse mean value inequality.

Lemma 2.1. Let $h(t)$ be a nonnegative and monotone decreasing function on the interval $0 \leq x \leq b$. If there exists a constant $D>1$ such that

$$
\frac{1}{s} \int_{0}^{s} h(t) d t \leq D h(s)
$$

for every $0 \leq s \leq b / 20$, then

$$
\int_{0}^{b}|h(t)|^{r} d t \leq \frac{20^{r} b^{1-r} D}{D-r(D-1)}\left(\int_{0}^{b} h(t) d t\right)^{r}
$$

for any $1 \leq r \leq D /(D-1)$.

Using Lemmas 1.1 and 2.1 we prove the following:

Theorem 2.1. Let $f$ be a nonnegative function belonging to $L_{\mathrm{loc}}^{1}\left(Q_{0}\right)$ and satisfying condition (WR) with $w$ satisfying condition $(D)$. Then $f$ belongs to $L_{\mathrm{loc}}^{r}\left(Q_{0}\right)$, for any $p \leq r<p+\varepsilon$, where $\varepsilon$ is a positive constant depending on $K, p, w$, and $n$.

Proof. Put $g(x)=\left(M f+f^{\sharp}\right)^{p}(x)$ for any $x \in \bar{Q}$. From Lemma 1.1 we deduce that $g(x)$ is an $A_{1}$-weight. Then for any $x \in \bar{Q}$ we have

$$
M g(x) \leq D g(x)
$$

with $D=c K, c$ a positive constant depending on $n, p, w$.

We indicate by $h^{*}(t)$ the decreasing rearrangement of $h$ with respect to the measure $\mu$ (see $[5,14,15]$, e.g.), and we deduce from (2.1) the inequality

$$
(M g)^{*}(t) \leq D g^{*}(t)
$$

for any $0<t<\mu(\bar{Q})$. Using the weighted Herz theorem (see [5, 14] e.g.)

$$
\frac{1}{t} \int_{0}^{t} g^{*}(s) d s \leq C(M g)^{*}(t)
$$

and then

$$
\frac{1}{t} \int_{0}^{t} g^{*}(s) d s \leq C D g^{*}(t)
$$

for any $0<t<\mu(\bar{Q})$, where $C$ depends only on $n$ and the constant $d$ appearing in condition $(D)$.

From (2.2), using Lemma 2.1 we deduce

$$
\int_{0}^{\mu(\bar{Q})}\left|g^{*}(t)\right|^{r} d t \leq \frac{20^{r} \mu(\bar{Q})^{1-r} C D}{C D-r(C D-1)}\left(\int_{0}^{\mu(\bar{Q})} g^{*}(t) d t\right)^{r}
$$

for any $1 \leq r<C D /(C D-1)$, from which

$$
\int_{\bar{Q}}\left(M f+f^{\sharp}\right)^{p r}(x) d \mu \leq \frac{20^{r} \mu(\bar{Q})^{1-r} C D}{C D-r(C D-1)}\left(\int_{\bar{Q}}\left(M f+f^{\sharp}\right)^{p}(x) d \mu\right)^{r},
$$


for any $1 \leq r<C D /(C D-1)$. Then, using Hardy-Littlewood and FeffermanStein maximal inequalities, (see [2] e.g.), $f$ belongs to $L^{p r}(\bar{Q})$ for $1 \leq r<$ $C D /(C D-1)$ and the theorem is proved.

We note that condition (WR) is not immediately comparable with the reverse Hölder inequality $[3,5,10,12,14,16]$

$$
\left(f_{\sigma Q}|f|^{p} d \mu\right)^{1 / p} \leq K f_{Q}|f| d \mu .
$$

Moreover, in the significant cases $K>1,0<\varepsilon<1$, condition (WR) and the Gurov-Reshetnyak condition [3, 9, 14]

$$
\left(f_{\sigma Q}\left|f-f_{\sigma Q} f d \mu\right|^{p} d \mu\right)^{1 / p} \leq \varepsilon f_{\sigma Q}|f| d \mu
$$

are not related.

\section{REFERENCES}

1. C. Bennett and R. Sharpley, Weak-type inequalities for $H^{p}$ and BMO, Proc. Sympos. Pure Math., vol. 35, Amer. Math. Soc., Providence, R.I., 1979, pp. 201-229.

2. _ Interpolation of operators, Academic Press, New York, 1988.

3. B. Boyarski, On Gurov-Reshetnyak classes (to appear).

4. B. Boyarski and T. Iwaniec, Analytical foundations of the theory of quasiconformal mappings in $R^{n}$, Ann. Acad. Sci. Fenn. Ser. A 1 Math. 8 (1983), 257-324.

5. A. Canale, $A$ higher integrability theorem from reverse Hölder weighted inequalities with different supports, Boll. Un. Mat. Ital. (to appear).

6. R. Coifman and C. Fefferman, Weighted norm inequalities for maximal functions and singular integrals, Studia Math. 51 (1974), 241-250.

7. E. Fabes, C. E. Kenig, and R. Serapioni, The local regularity of solutions of degenerate elliptic equations, Comm. Partial Differential Equations 7 (1982), 77-116.

8. M. Franciosi, Maximum principle for second order elliptic equations and applications, J. Math. Anal. Appl. (to appear).

9. __ Weighted rearrangements and higher integrability results, Studia Math. 92 (1989), 31-39.

10. M. Franciosi and G. Moscariello, Higher integrability results, Manuscripta Math. 52 (1985), 151-171.

11. _ $A$ note on the maximum principle for 2 nd order non variational linear elliptic equations, Ricerche Mat. XXXV (1986), 279-290.

12. F. W. Gehring, The $L^{p}$-integrability of the partial derivatives of a quasiconformal mapping, Acta Math. 130 (1973), 265-277.

13. C. Herz, The Hardy-Littlewood maximal theorems, Sympos. on Harm. Analysis, Univ. of Warwick, 1968.

14. T. Iwaniec, On $L^{p}$ integrability in PDE's and quasiregular mappings for large exponents, Ann. Acad. Sci. Fenn. Ser. A 1 Math. 7 (1982), 301-332.

15. B. Muckenhoupt, Weighted inequalities for the Hardy maximal function, Trans. Amer. Math. Soc. 165 (1972), 207-226.

16. C. Sbordone, Higher integrability from reverse integral inequalities, methods of functional analysis and theory of elliptic equations, Napoli, 1982, pp. 347-357, Liguori, Napoli, 1983. 
17. Un. Mat. Ital. C (6) 1 (1986), 73-94.

18. __ Some reverse integral inequalities, Atti Accad. Pontaniana, Napoli, 1984.

19. I. Wik, Note on a theorem by Reshetnyak-Gurov, Studia Math. 87 (1987), 287-290.

20. __ On Muckenhoupt's classes of weight functions, Studia Math. Instituto Di Matematica, Barorllssi, 84100 Salermo (to appear).

Istituto di Matematica, Università di Salerno, Baronissi, 84110, Salerno, Italy 